New Ambassadors appointed by the College of General Dentistry

The College of General Dentistry (CGDent) has announced the appointment of two new Ambassadors: Dr Wendy Thompson and Dr Yewande Oduwole.

Those Ambassadors of the College who are dental healthcare professionals are drawn from across the spectrum of dentistry. They support the mission and vision of the College and encourage inclusive professional engagement in the development of the College. Ambassadors help the College promote dentistry as an important element of general healthcare and wellbeing. They support the College's mission to promote life-long, preventatively orientated, minimum intervention, personalised, holistic dental care.

More Ambassadors will be appointed in the coming months as the College works towards its historic, formal launch, rescheduled for early 2021, COVID restrictions permitting.

Chair of the College Board of Trustees, Professor Nairn Wilson, said: 'I am delighted that both Wendy and Yewande have accepted the invitation to become Ambassadors for the College. With their help and support, it is hoped to engage many more early career members of the dental team in the further development of the College, which, as key elements of its mission, aims to provide all dental healthcare professionals with new leadership and much-needed career pathways. The Board of Trustees looks forward to working with Wendy and Yewande in realising the College goal of being valued by a large diverse, inclusive membership'

\section{Storytelling}

Shaun Sellars continues this series on ethical dilemmas in dentistry which appears in every second issue of the $B D J$.

\section{Up until recently, I wasn't a great supporter of} community water fluoridation (CWF). Looking at the evidence, the case for routine CWF didn't appear to be compelling. A small benefit to a limited cohort, mainly based on older, outdated research didn't override the concerns I had over the paternalistic approach that CWF takes.

But in the last year or so, two things have changed. Firstly, I moved from Lincoln to Suffolk. Lincoln is a city which receives fluoridated water, and in the practice I was working at, I barely saw any children with caries. Suffolk, however, is an area without CWF and the decay is visible and problematic. The effect of caries on children and their parents has become all too evident and considering the social demographics of the area I've worked in hasn't changed significantly, I can only assume the lack of fluoridation is a significant contributory factor.

The other reason I've changed my mind is thanks to a conversation I had with the head of a social enterprise which provides community dental services. The topic of fluoridation came up, and we were discussing the evidential and ethical pros and cons. The Damascene moment which changed my mind suddenly concerned the dental health of the homeless. To paraphrase, 'sometimes the only dental care homeless people will have access to is from fluoridated water.' My ivory tower collapsed instantaneously.

Both of these reasons have the same root cause: a lack of insight. In Lincoln, I rarely saw children with caries, so I underestimated the severity of the problem and the knock-on effect it has on families. Despite knowing the statistics on caries in children, it's taken the physical act of seeing patients in distress to overcome my pre-existing aversion to CWT. And the plight of the homeless, from a dental perspective, was something that I'd taken little time to consider previously. Because I wasn't aware of their situation, I couldn't consider it in my assessment of the value of CWT. Once this was made apparent to me, I could look at the situation with an expanded sense of understanding. It took these stories I'd seen unfold to make me reconsider my position.

I'm seeing similar with patients. Fortunately, Suffolk fared relatively well through the coronavirus outbreak. Patients routinely comment that they don't know anyone who's died or even been diagnosed with COVID-19. There's often some scepticism of the severity of the problem. Unfortunately, we know family members of patients who have died as a result of COVID-19. When you take time to explain the realities of the disease, there's a similar re-evaluation of the problem.

At the moment, it feels as if we're living in a world of increasingly entrenched and extreme ideas and values. Levels of intellectual conflict are high, and people are more unwilling than ever to change their viewpoints based on new information. Dentists and dentistry are not immune to this. Facts and figures don't change minds, stories do. And when they're supported by evidence, they can be transformative.

\title{
BAOMS welcomes call for ring-fenced hospital beds
}

The British Association of Oral and Maxillofacial Surgeons (BAOMS) has welcomed the call from the Royal College of Surgeons of England (RCSEng) to ring-fence hospital beds for planned operations to avoid future mass cancellations during a second wave of COVID-19.
BAOMS says oral and maxillofacial (OMFS) units across the UK have limited access to theatres for both elective and semielective (facial trauma) cases and are well short of the target of $90 \%$ elective surgical capacity for routine operations. This does not affect OMFS cancer patients. OMFS fear that an increase in COVID cases could lead to the cancellation of all normal activity. Rob Bentley, BAOMS President, said: 'We have learnt ways to work with COVID safely and provide elective surgery for our patients. If we create "protected" theatre and beds we can start to address the enormous backlog generated by the first wave'. 